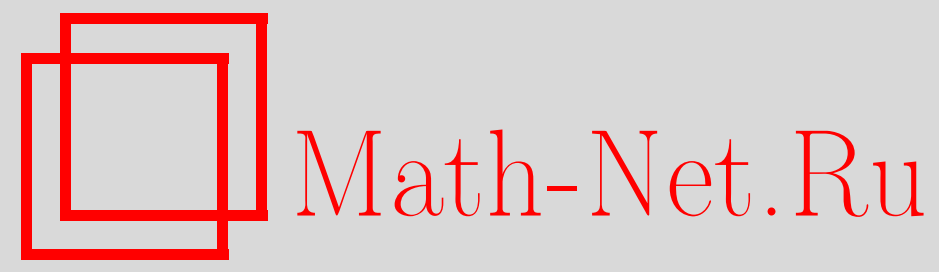

В. Р. Фаталов, Большие уклонения гауссовских мер в пространствах $l^{p}$ и $L^{p}, p \geq 2$, Теория вероятн. и ее примен., 1996, том 41, выпуск 3, 682-689

DOI: https://doi.org/10.4213/tvp3183

Использование Общероссийского математического портала Math-Net.Ru подразумевает, что вы прочитали и согласны с пользовательским соглашением http://www . mathnet.ru/rus/agreement

Параметры загрузки:

IP : 54.81 .137 .203

26 апреля 2023 г., $16: 20: 42$

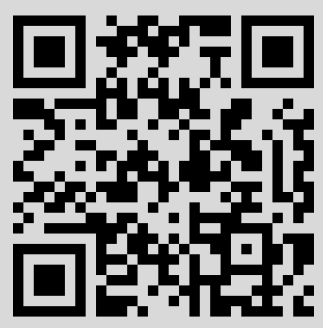




\section{СПИСОК ЛИТЕРАТУРЫ}

1. Гихмая И. И., Скороход А. В. Теория случайных процессов. Т. 1. М.: Наука, $1971,664 \mathrm{c}$.

2. Радченко B. Н. Теорема Радона-Никодима для случайных мер. - Укр. матем. журн., 1989, т. 41, № 1, с. 63-67.

Поступила в редакцию

14.III.1992

(C) $1996 \mathrm{r}$.

ФАТАЛІОВ В. P.*

\section{БОЛЬШИЕ УКЛОНЕНИЯ ГАУССОВСКИХ MEP B IPOCTPAHCTBAX $l^{p}$ И $L^{p}, p \geqslant 2$}

В настоящее время интенсивно развивается теория суммирования независимых случайных элементов со значениями в банаховом пространстве. Вследствие центральной предельной теоремы в качестве предельных возникают гауссовские распределения. В данной работе найдены точные асимптотики больших уклонений гауссовских мер множеств в обших банаховых пространствах. Полученный в статье основной результат применен для вычисления асимптотик гауссовских мер шаров в пространствах $l^{p}$ и $L^{p}, p \geqslant 2$ (в случае винеровской меры). Обсуждаются приложения к теории статистик типа $\omega^{p}, p \geqslant 2$.

Ключевые слова и Фразы: гауссовские меры в банаховых пространствах, большие уклонения, пространства $l^{p}$ и $L^{p}, p \geqslant 2$, винеровская мера, $\omega^{p}$-статисти$\mathbf{k} \mathbf{a}, p \geqslant 2$.

1. Основной результат. Пусть $E$ - вешественное сепарабельное банахово пространство с нормой $\|\cdot\|, E^{*}-$ его топологическое сопряженное. Пусть $P_{A}-$ гауссовская вероятностная мера, определенная на борелевской $\sigma$-алгебре подмножеств в $E$, со средним нуль и ковариационным оператором $A$. В данной работе речь пойдет о точной асимптотике вероятности $P_{A}(u D), u \rightarrow \infty$, для некоторого борелевского множества $D$.

Как известно, ковариационный оператор $A$ является симметричным и положительным $[1$, с. 120$]$ ядерным $[1$, с. 143$]$ оператором, отображающим $E^{*}$ в $E$. Предположим, что $A$ инъективен, т.е. $A x^{*}=0$ тогда и только тогда, когда $x^{*}=0$. Тогда область значений $R(A)=\left\{A x^{*}: x^{*} \in E^{*}\right\}$ оператора $A$ всюду плотна в $E[1$, с. 122]. Через $\left\langle x, x^{*}\right)$ будем обозначать значение функционала $x^{*} \in E^{*}$ в точке $x$. Далее, пусть выполнены следующие три условия

(C1) Борелевское множество $D$ замкнуто и допускает представление $D=$ $\{x \in E: G(x) \geqslant 0\}$, әде $G(x)$ - такая мепрерывкая вещественяозначная фунхиия, что или $D$ или мкожество $E \backslash D$ представимо в виде конечного объединекия непересекающихся выпукльх жножеств из $E$. Iредположик также, ито $D$ имеет кепустую вяутреняость и $D$ ке содержит нулевого элемента из $E$.

(C2) Квадратическая форма $\left\langle x, A^{-1} x\right\rangle$ достигает своего минимума на $D$ в конечном числе точех $x^{1}, x^{2}, \ldots, x^{m}$ из араницы множества $D$. (Если $x$ не прияадлежит области определекия $\operatorname{Dom}\left(A^{-1}\right)$ оператора $A^{-1}$, то мы считаем $\left\langle x, A^{-1} x\right\rangle=$

*Ереванский государствениый университет, Межвузовский научный Центр по прикладным проблемам математики, ул. Алека Манукяна, 1, 375049 Ереван, Армения. 
$+\infty$ - это несучествекно для кашего изложения, так как $R(A)$ всюду плотко в $E$ ).

(С3) Функиия $G$ дважды дифференцируема по Фреше в окрестностях точек $x^{1}, \ldots, x^{m}$ с производными $G^{\prime}(x), G^{\prime \prime}(x)$, причем $\left\langle x^{i}, G^{\prime}\left(x^{i}\right)\right\rangle \neq 0, i=1,2, \ldots, m$. Пусть, далее, операторы $G^{\prime \prime}\left(x^{i}\right)$ инъективны, $i=1,2, \ldots, m$. Предположим, что собстееняые значекия $\mu_{k}^{i}, k=1,2, \ldots, M_{i}, M_{i} \leqslant \infty$, оператора $A G^{\prime \prime}\left(x^{i}\right)$ таковы, что определен и комечен детермияант

$$
0<\operatorname{det} B_{i}=\prod_{k=1}^{M_{i}}\left(1-\lambda_{i} \mu_{k}^{i}\right), \quad i=1,2, \ldots, m,
$$

әде $B_{i}=I-\lambda_{i} A G^{\prime \prime}\left(x^{i}\right), \lambda_{i}=\left\langle x^{1}, A^{-1} x^{1}\right\rangle /\left\langle x^{i}, G^{\prime}\left(x^{i}\right)\right\rangle, I-$ тождестөенный операmop uз $E$ в $E$.

3 а м е ч а н и я. 1. Напомним, что $G^{\prime}(x)$ и $G^{\prime \prime}(x)$ являются ограниченными линейными операторами из $E$ в $\mathbf{R}^{1}$ и из $E$ в $E^{*}$ соответственно. Поскольку множество всех ядерных операторов, действующих на всем классе банаховых пространств, является операторным идеалом [2, с. 99], то оператор $A G^{\prime \prime}\left(x^{i}\right)$-ядерный, отображающий $E$ в $E$. Следовательно, $A G^{\prime \prime}\left(x^{i}\right)$ - компактный оператор и имеет не более чем счетное множество собственных значений $\mu_{k}^{i}$. Детерминант (1), очевидно, конечен, если сходится рхд $\sum_{k=1}^{\infty}\left|\mu_{k}^{i}\right|$. В отличие от случая гильбертова пространства одной ядерности отератора $A G^{\prime \prime}\left(x^{i}\right)$ для этого, вообще говоря, недостаточно [2, с. 445] требуется чуть большее: например, принадлежность $A G^{\prime \prime}\left(x^{i}\right)$ классу $\mathfrak{N}_{(1,2,1)}(E, E)$ (см. $[2$, с. 445]).

2. Несложно показать, что

$$
\inf \left\{\left\langle x, A^{-1} x\right\rangle: x \in D\right\}=\frac{1}{\sup \left\{\left\langle A x^{*}, x^{*}\right\rangle: x^{*} \in D^{\circ}\right\}},
$$

где $D^{\circ}=\left\{x^{*}: \sup _{x \in D}\left\{\left\langle x, x^{*}\right\rangle \leqslant 1\right\}\right.$ есть поляра множества $D$.

В работе [3] дохазан следуюший результат

Теорема 1. Пусть все сФормулированные выне предположения, включая условия (C1)-(С3), выполнекы. Тогда верно следуючее асимптотическое соотночекие

$$
\begin{aligned}
P_{A}(u D)= & \exp \left\{-\frac{u^{2}}{2}\left\langle x^{1}, A^{-1} x^{1}\right\rangle\right\} \frac{1}{u \sqrt{2 \pi}} \\
& \times \sum_{i=1}^{m}\left|\operatorname{det} B_{i}\left\langle B_{i}^{-1} x^{i}, A^{-1} x^{i}\right\rangle\right|^{-1 / 2}(1+o(1)), \quad u \rightarrow \infty,
\end{aligned}
$$

әде $B_{i}^{-1}$ есть обратный оператор $\times B_{i}$.

3 а м е ч а н и е. Если функция $G$ дифференцируема по Фреше три раза, то $o(1)$ в формуле (2) можно заменить на $O\left(u^{-2}\right)$ (см. также теорему 3 ниже).

Теорема 1 может найти применение в теории суммирования независимых случайных вехторов в банаховом пространстве, (см. [4], [5]), где гауссовское распределение появляется в качестве предельного. $p \geqslant 2$.

2. Гауссовские большие уклонения норм в пространствах $l^{p}$ и $L^{p}$,

Условия теоремы 1 выполнены в пространствах $l^{p}, L^{p}[0,1] \equiv L^{p}, p \geqslant 2$, для функций $G(x)$, равных нормам в этих пространствах. Последние дважды дифференцируемы по Фреше только при $p \geqslant 2$ [6]. Рассматриваемые ниже случайные элементы заданы на некотором вероятностном пространстве $[\Omega, \mathcal{F}, \mathbf{P}]$.

Теорема 2. Пусть $\left(X_{1}, X_{2}, \ldots\right)$ - гауссовский элемент в $l^{p}, p>2$, имеюичй кулевое среднее и невырождеяную ковариачиояную матричу $A=\left(a_{i j}\right), a_{i j}=$ 
$\mathrm{E} X_{i} X_{j}, i, j=1,2, \ldots$, см. $\left[1\right.$, с. 261]. Пуусть $z^{1}, z^{2}, \ldots, z^{m}-$ точки из множества $Z=\left\{x=\left(x_{1}, x_{2}, \ldots\right) \in l^{p}: \sum_{j=1}^{\infty}\left|x_{j}\right|^{p}=1\right\}$, в которых достигается минимум $\min \left\{\left\langle x, A^{-1} x\right\rangle: x \in Z\right\}=1 / \sigma^{2}>0, z^{i}=\left(z_{i 1}, z_{i 2}, \ldots\right), i=1, \ldots, m$.

Тогда имеет место соотноиение

$$
\begin{aligned}
\mathbf{P}\left\{\left(\sum_{j=1}^{\infty}\left|X_{j}\right|^{p}\right)^{1 / p}>u\right\}= & \exp \left\{-\frac{u^{2}}{2 \sigma^{2}}\right\} \frac{\sigma}{u \sqrt{2 \pi}} \sqrt{p-2} \\
& \times \sum_{i=1}^{m}\left|\operatorname{det} B_{i}\right|^{-1 / 2}(1+o(1)), \quad u \rightarrow \infty,
\end{aligned}
$$

при условии, что $0<\left|\operatorname{det} B_{i}\right|<\infty$, гдe

$$
B_{i}=I-(p-1) \sigma^{-2} A \operatorname{diag}\left(\left|z_{i 1}\right|^{p-2},\left|z_{i 2}\right|^{p-2}, \ldots\right), \quad i=1,2, \ldots, m .
$$

Здесь ниже $\operatorname{diag}\left(c_{1}, c_{2}, \ldots\right)$ обозначает диагональную матрицу с элементами $c_{1}$, $c_{2}, \ldots$ по главной диагонали, матричное представление линейных операторов соответствует выбранному базису.

В условиях теоремы 2 в силу замечания 2 имеют место соотношения

$$
\begin{gathered}
\sigma^{-2}=\inf \left\{\left\langle x, A^{-1} x\right\rangle: x \in Z\right\}=\left(\sup \left\{\left\langle A x^{*}, x^{*}\right\rangle:\left\|x^{*}\right\|_{l^{q}} \leqslant 1\right\}\right)^{-1}=\|A\|^{-1}, \\
p^{-1}+q^{-1}=1, \quad\|x\|_{l^{p}}=\left(\sum_{i=1}^{\infty}\left|x_{i}\right|^{p}\right)^{1 / p} .
\end{gathered}
$$

3 а м е ч а н и е 3 . Результат, аналогичный теореме 2 , для $p=2$ был получен ранее В. М. Золотаревым в [7] методом преобразований Лапласа.

Следствие 1. Пусть $\left(X_{1}, X_{2}, \ldots\right)$ - аауссовский элемент в $l^{p}, p^{p}>2$, со среджим куль и мезависимыми коордияатами, $\mathrm{E} X_{i} X_{j}=0, i \neq j, \mathrm{E} X_{i}^{2}=\sigma_{i}^{2}>0$, $i, j=1,2 \ldots, A=\operatorname{diag}\left(\sigma_{1}^{2}, \sigma_{2}^{2}, \ldots\right)$, причем $\sigma_{1}^{2}=\sigma_{2}^{2}=\cdots=\sigma_{k}^{2}>\sigma_{k+1}^{2} \geqslant \cdots$. Toгда минимум вьражекия $\left\langle x, A^{-1} x\right\rangle$ жа мможестве $\left\{x \in l^{p}:\|x\|_{l p}=1\right\}$ равен $\sigma_{1}^{-2}$ u достигается о $2 k$ mочках $z_{+}^{i}=(0, \ldots, 0,1,0, \ldots)$ (еटинича яа $i-\mu$ месте), $z_{-}^{i}=$ $(0, \ldots, 0,-1,0, \ldots)(-1$ на $і$-м месте $), i=1,2, \ldots, 2 k$.

Справедливо асимптотическое соотночение

$$
\mathbf{P}\left\{\left(\sum_{i=1}^{\infty}\left|X_{j}\right|^{p}\right)^{1 / p}>u\right\}=\exp \left\{-\frac{u^{2}}{2 \sigma_{1}^{2}}\right\} \frac{2 k \sigma_{1}}{\sqrt{2 \pi} u}(1+o(1)), \quad u \rightarrow \infty .
$$

Главный член асимптотики в формуле (4) ранее был найден разными методами (отличными от нашего) в [8] для $p>3$ и в [9] для $p>2$, без оценки остаточного члена. Асимптотики норм гауссовских элементов в конечномерных пространствах $l_{n}^{p}, p>0$, вычислены в [10] (случай независимых координат) и [11] (зависимые координаты).

Теорема 3. Пусть $W$ u $W_{0}$ - распределения викеровского прочесса $w(t)$ и броуновского моста $\stackrel{\circ}{u}(t)=w(t)-t w(1)$ в $L^{p}, p \geqslant 2\left(W u W_{0}\right.$, как известно, сосредоточекы ма подпространстве $C[0,1]$ непрерывных ма $[0,1]$ фуккичй). Тогда:

$$
\begin{aligned}
& \mathbf{P}\left\{\left(\int_{0}^{1}|w(t)|^{p} d t\right)^{1 / p}>u\right\}=W\left\{x \in L^{p}:\|x\|_{L^{p}}>u\right\} \\
& =\exp \left\{-\frac{u^{2}}{2 \sigma^{2}}\right\} \frac{2 \sigma}{u} \pi^{-3 / 4}\left(\frac{\Gamma\left(\frac{1}{2}+1 / p\right)}{\Gamma(1+1 / p)}\right)^{1 / 2}(1+o(1)), \quad u \rightarrow \infty,
\end{aligned}
$$


где $\sigma=[2 /(\pi p)]^{1 / 2}(2+p / 2)^{(p-2) /(2 p)} \Gamma\left(\frac{1}{2}+1 / p\right) / \Gamma(1+1 / p), \Gamma(\cdot)-$ гамма-фуякчия, $\|x\|_{L^{p}}=\left(\int_{0}^{1}|x(t)|^{p} d t\right)^{1 / p}$

(ii) $\mathbf{P}\left\{\left(\int_{0}^{1}|\stackrel{o}{u(t)}|^{p} d t\right)^{1 / p}>u\right\}=W_{0}\left\{x \in L^{p}:\|x\|_{L^{p}}>u\right\}$

$$
=\exp \left\{-\frac{2 u^{2}}{\sigma^{2}}\right\} \frac{\sigma}{u} \pi^{-3 / 4}\left(\frac{\Gamma\left(\frac{1}{2}+1 / p\right)}{\Gamma(1+1 / p)}\right)^{1 / 2}(1+o(1)), \quad u \rightarrow \infty,
$$

где $\sigma$ mо же, ито и в пункте (i).

Для пространства $C[0,1]^{n}$ и нормы супремума в нем (которая всего лишь субдифференцируема) аналогичные асимптотики для распределений гауссовских неоднородных полей (в том числе, вознихающих в статистике) вычислены иным методом B [12].

3. Доказательства. Подробное доказательство теоремы 1 , основанное на работах [13]-[15] (см. также [16]), приведено в [3]. Здесь мы докажем теоремы 2 и 3. Из результатов статьи [17] следует, что в утверждения теорем 2 и 3 неравенства под знаком вероятности могут быть строгими.

Пусть $H=H(A) \subset E$ - гильбертово пространство, ассоциированное с ковариащионным оператором $A$ и нормой $\|\cdot\|_{A}$ (см. $[1$, с. 126]). Тогда, как хорошо известно, $[14$, с. 34$]$, функционал действия $I(x)$ гауссовской меры $P_{A}$ определяется так:

$$
I(x)=\left\{\begin{array}{lll}
\frac{1}{2}\|x\|_{A}^{2}, & \text { если } & x \in H \\
+\infty, & \text { если } & x \in E \backslash H
\end{array}\right.
$$

Решая экстремальную задачу $I(x) \rightarrow \inf$ при ограничении $G(x) \geqslant 0$ методом множителей Лагранжа, получаем для экстремальных точек $x^{i}$ из условия (C2):

$$
\begin{aligned}
x^{i} & \in \operatorname{Dom}\left(A^{-1}\right), \\
G\left(x^{i}\right) & =0, \quad A^{-1} x^{i}=\lambda_{i} G^{i}\left(x^{i}\right)
\end{aligned}
$$

(рассуждения, приведенные в [11, лемма 1] для случая гильбертова пространства $E$, применимы и в нашем случае).

Перейдем теперь непосредственно к доказательству теорем.

Вывод формулы (3) из (2) совсем прост. Пусть $\left\{e_{k}\right\}$ - стандартный базис в $l^{p}$. Функция $G(x)=\sum_{k=1}^{\infty}\left|x_{k}\right|^{p}-1, x=\sum_{k=1}^{\infty} x_{k} e_{k}$, дважды дифференцируема по Фреше при $p>2[6$, с. 887], с производными:

$$
G^{\prime}(x)=\left(p\left|x_{k}\right|^{p-1} \operatorname{sign} x_{k}\right)_{k=1}^{\infty} \in l^{q}, \quad G^{\prime \prime}(x)=p(p-1) \operatorname{diag}\left(\left|x_{1}\right|^{p-2},\left|x_{2}\right|^{p-2}, \ldots\right),
$$

$p^{-1}+q^{-1}=1$. Используя (5), находим для точки минимума $z^{i}=\sum_{k=1}^{\infty} z_{i k} e_{k}: \lambda_{i}=$ $p / \sigma^{2}, i=1, \ldots, m$. Поскольху $G^{\prime \prime}(x) x=(p-1) G^{\prime}(x)$, то, вновь используя (5), имеем $B_{i} z^{i}=(2-p) z^{i}$, откуда $\left\langle B_{i}^{-1} z^{i}, A^{-1} z^{i}\right\rangle=\sigma^{-2} /(2-p), i=1, \ldots, m$.

Д ок а з а т е л с с т в о т е о е м ы 3 проведем для случая (i) винеровской меры, пункт (ii) рассматривается аналогично.

а) Решение экстремальной задачи для винеровской меры в $L^{p}[0,1]$. Пусть сначала $p>2$. Обозначим $G(x)=\int_{0}^{1}|x(t)|^{p} d t-1$ для $x \in L^{p}$. Эта функция дважды дифференцируема по Фреше с производными (см. [6, с. 887]):

$$
G^{\prime}(x)=p|x(t)|^{p-1} \operatorname{sign} x(t) \in L^{q}, \quad G^{\prime \prime}(x)[y]=p(p-1)|x(t)|^{p-2} y(t) \in L^{q},
$$


$x, y \in L^{p}, 1 / p+1 / q=1$. Ковариационный оператор $A$ винеровской меры $W$ отображает $L^{q}$ в $L^{p}$ по правилу (см. $[13$, с. 47$],[18]$ ):

$$
A x(t)=\int_{0}^{1} \min (t, s) x(s) d s, \quad x(s) \in L^{q}
$$

Уравнение (5) для экстремальной функции $x(t)$ принимает вид

$$
x(t)=p \lambda \int_{0}^{1} \min (t, s)|x(s)|^{p-1} \operatorname{sign} x(s) d s, \quad\|x\|_{L^{p}}=1 .
$$

Здесь $x \in \operatorname{Dom}\left(A^{-1}\right)$, где $A^{-1}=-d^{2} / d t^{2}$, и, следовательно, экстремальная функция $x(t)$ дважды дифференцируема. Интегральное уравнение (Хаммерштейна) (6) легко приводится к дифференциальному

$$
\left\{\begin{array}{l}
x^{\prime \prime}(t)=-\lambda p|x(t)|^{p-1} \operatorname{sign} x(t) \\
x(0)=x^{\prime}(1)=0
\end{array}\right.
$$

Записывая первое равенство в (7) в виде $d\left(x^{\prime}(t)\right)^{2}=-\lambda d|x(t)|^{p}$, получаем: $\left(x^{\prime}(t)\right)^{2}=$ $2 \lambda\left(|x(1)|^{p}-|x(t)|^{p}\right), t \in[0,1]$. Отсода видно, что $|x(t)| \leqslant|x(1)|$ для всех $t \in[0,1]$. Поскольку $x^{\prime}(t)$ - непрерывная функция, то возможны два решения уравнения (7): $x_{1}(t)$ с $x_{1}^{\prime}(t)=\sqrt{2 \lambda} \sqrt{\left.\left|x_{1}(1)\right|^{p}-\left|x_{1}(t)\right|^{p}\right)} \geqslant 0, x_{1}^{\prime}(t)$ монотонно возрастает, $x_{1}(t) \geqslant 0$ и $x_{2}(t)=-x_{1}(t), t \in[0,1]$. Итак, имеем дифференциальное уравнение (нижний индекс 1 опущен):

$$
\left\{\begin{array}{l}
x_{1}^{\prime}(t)=\sqrt{2 \lambda} \sqrt{\left.|x(1)|^{p}-|x(t)|^{p}\right)}, \\
x(0)=0 .
\end{array}\right.
$$

Отсюда получаем: $\int_{0}^{x}\left(C-x^{p}\right)^{-1 / 2} d x=\sqrt{2 \lambda} t, C=(x(1))^{p}$. Используя равенство под номером 4 из $[19$, с. 30$]$, приходим к соотношению

$$
\frac{x(t)}{\sqrt{C}} F\left(\frac{1}{2}, \frac{1}{p} ; 1+\frac{1}{p} ; \frac{(x(t))^{p}}{C}\right)=\sqrt{2 \lambda t},
$$

где $F(a, b ; c ; z)$ обозначает гипергеометрическую функцию Гаусса. Взяв здесь $t=1$, найдем

$$
(x(1))^{p / 2-1}=\sqrt{\frac{\pi}{2 \lambda p}} \frac{\Gamma(1 / p)}{\Gamma(1 / p)+\frac{1}{2}} .
$$

Переходя теперь к функциям Лежандра $P_{\nu}^{r}(t)$ по формуле 15.4 .13 из справочника [20, c. 376] и выражая $\lambda$ через $C$, получаем из (9) основное функциональное уравнение для экстремальной функции $x(t)$ :

$$
2^{1 / p}\left(\frac{x(t)}{x(1)}\right)^{1 / 2} P_{-1 / p}^{-1 / p}\left(\sqrt{1-\left(\frac{x(t)}{x(1)}\right)^{p}}\right)=\sqrt{\pi} t
$$

Вычислим теперь константы $C$ и $\sigma^{2}$. Из (8) имеем $C=1 /(2 \lambda) \int_{0}^{1}\left(x^{\prime}(t)\right)^{2} d t+1$, но, в силу (7), $\int_{0}^{1}\left(x^{\prime}(t)\right)^{2} d t=-\int_{0}^{1} x(t) x^{\prime \prime}(t) d t=\lambda p$. Следовательно, $C=1+p / 2$. Далее, с учетом равенства (5) и вида производной $G^{\prime}(x)$ имеем $\sigma^{-2}=\left\langle x, A^{-1} x\right\rangle=$ $\lambda\left\langle x, G^{\prime}(x)\right\rangle=\lambda p$. Теперь формула (10) позволяет найти

$$
\sigma^{-2}=\frac{\pi}{2 p}\left(1+\frac{p}{2}\right)^{(2-p) / p}\left(\frac{\Gamma(1 / p)}{\Gamma\left(\frac{1}{2}+1 / p\right)}\right)^{2} .
$$


в) Вычисление определителя $\operatorname{det}\left(I-\lambda A G^{\prime \prime}(x)\right)$. Найдем собственные значения $\mu_{k}$ оператора $A G^{\prime \prime}(x)$, соответствуюшие собственным функциям $z_{k}$. Для этого рассмотрим уравнение (относительно $\mu$ и $z$ ): $A G^{\prime \prime}(x) z=\mu z, z \in L^{p}$. Это уравнение равносильно дифференциальному уравнению

$$
\left\{\begin{array}{l}
p(p-1)(x(t))^{p-2} z(t)=-\mu z^{\prime \prime}(t) \\
z(0)=z^{\prime}(1)=0
\end{array}\right.
$$

Легко видеть, что уравнение (11) имеет частное решение $z_{1}(t)=x(t)$ с собственным числом $\mu_{1}=(p-1) / \lambda$. Замена $s=(x(t) / x(1))^{p}$ приводит уравнение (11) к гипергеометрическому уравнению для функции $y(s)=z(t)$ :

$$
s(s-1) y^{\prime \prime}(s)+\left(1-\frac{1}{p}-\left(\frac{3}{2}-\frac{1}{p}\right) s\right) y^{\prime}(s)+\frac{p-1}{2 \lambda \mu p} y(s)=0, \quad y(0)=0 .
$$

Анализ этого дифференциального уравнения показывает, что здесь возможен только случай В из $\left[20\right.$, с. 377], когда собственные числа равны $\mu_{k}=(p-1) /\left(2 \lambda p\left(k-\frac{1}{2}\right)(k-\right.$ $1+1 / p)), k=1,2, \ldots$, a соответствующие собственные функции выражаются через полиномы Якоби (см. также таблицу на с. 83 в [21], случай 4):

$$
z_{k}(t)=x(t) F\left(1-k, k+\frac{1}{p}-\frac{1}{2} ; 1+\frac{1}{p} ;\left(\frac{x(t)}{x(1)}\right)^{p}\right), \quad k=1,2, \ldots
$$

Наконец, используя № 11 из [19, с. 754], находим

$$
\operatorname{det}\left(I-\lambda A G^{\prime \prime}(x)\right)=\prod_{k=1}^{\infty}\left(1-\lambda \mu_{k}\right)=\sqrt{\pi}\left(\frac{p}{2}-1\right) \frac{\Gamma(1+1 / p)}{\Gamma\left(\frac{1}{2}+1 / p\right)} .
$$

Вычислим теперь последнюю константу в формуле (2). Поскольку $A G^{\prime \prime}(x) x=$ $\mu_{1} x$, то для оператора $B=I-\lambda A G^{\prime \prime}(x)$ имеем $B^{-1} x=(2-p)^{-1} x$. Следовательно, $\left\langle B^{-1} x, A^{-1} x\right\rangle=\sigma^{2} /(2-p)$. Вычисления для второй экстремальной функции $-x(t)$ аналогичны. Применяя формулу (2) из теоремы 1 , получаем утверждение (i) при $p>2$. Случай $p=2$ исследован в примере 1 в [11].

Доказательство пункта (ii) теоремы 3 осушествляется аналогично. Здесь оператор $A$ имеет ядро $\min (t, s)-t s$ и уравнение (7) остается прежним, но с иными граничными условиями: $\boldsymbol{x}(0)=x(1)=0$. Имеем две экстремальные функции $x(t)$ и $-x(t)$, функция $x(t) \geqslant 0$ имеет максимум в точке $t=\frac{1}{2}$ и симметрична относительно $t=\frac{1}{2}, x^{\prime}(t)>0, t \in\left[0, \frac{1}{2}\right)$. Теперь множитель Лагранжа $\lambda$ в 4 раза больше, чем в случае (i). Остальные рассуждения повторяют уже проведенные для меры $W$. Теорема 3 доказана.

4. Приложения к статистике и заключительные замечания. Пусть проверяется гипотеза $H_{0}$ о том, что выборка объема $n$ извлечена из распределения с непрерывной функцией распределения $F(t)$, через $F_{n}(t)$ обозначена эмпирическая функция распределения, построенная по выборке. Известно, $[22$, с. 196], что эмпирический процесс $\sqrt{n}\left(F_{n}(t)-F(t)\right)$ при гипотезе $H_{0}$ после замены $s=F(t)$ слабо сходится в $D[0,1]$ к броуновскому мосту $\stackrel{\circ}{w}(s)$. По аналогии со статистикой $\omega_{n}^{2}$ введем статистиху $\omega_{n}^{p}=\int_{0}^{1}(\sqrt{n})^{p}\left|F_{n}(t)-F(t)\right|^{p} d F(t)$. Тогда в силу слабой сходимости имеем $\omega_{n}^{p} \Longrightarrow \omega^{p}=\int_{0}^{1}|\dot{u}(s)|^{p} d s$, и теорема 3, п. (ii), позволяет найти приближенно вероятности $\lim _{n \rightarrow \infty} \mathbf{P}\left\{\omega_{n}^{p}>u_{\alpha}\right\}=\mathbf{P}\left\{\omega^{p}>u_{\alpha}\right\}=\alpha$ для больших значений $u_{\alpha}$. (малых $\alpha$ ) при $p \geqslant 2$. Отметим, однако, медленное (как правило, степенное) убывание поправочных членов. Статистики типа $\omega_{n}^{p}$ с весовыми функциями $q(t)$ рассмотрены в [4], [23]. Ясно, что результат типа теоремы 3 может быть получен на основе теоремы 1 и для функций $G(x)=\int_{0}^{1}|x(t)|^{p} q(t) d t-1, p \geqslant 2$. 
Наконец, отметим недавние работы [24]-[27], где методами, отличными от нашего, исследованы асимптотики больших уклонений гауссовских дважды дифференцируемых полунорм в бесконечномерных [24], [25], [27] и конечномерных [26] пространствах.

Автор выражает благодарность проф. В.-Д. Рихтеру (Ростовский университет) за полезные обсуждения при подготовке данной работы, а также статьи [11]. Автор признателен также рецензенту, чьи замечания способствовали улучшению данной публикацин.

\section{СПИСОК ЛИТЕРАТУРЫ}

1. Вахаяия Н. Н., Тариеладзе В. И., Чобанян С. А. Вероятностные распределения в банаховых пространствах. М.: Наука, 1985,368 c.

2. Пич $A$. Oператорные идеалы. М.: Мир, 1982, 536 с.

3. Fatalov V. R., Richter W.-D. Exact asymptotics for large deviations of Gaussian measures on Banach spaces. - Math. Nachr. (в печати).

4. Bentkus V.J., Račkauskas A. J. On probabilities of large deviations in Banach spaces. - Probab. Theory Relat. Fields, 1990, v. 86, № 2, p. 131-154.

5. Bolthausen $E$. Laplace approximations for sums of independent random vectors, I, II. - Probab. Theory Relat. Fields, 1986, v. 72, №. 2, p. 305-318; 1987, v. 76, № 2, p. 167-206.

6. Bonic R., Frampton J. Smooth functions of Banach manifolds. - J. Math. Mech., 1966 , v. 15 , № 5 , p. $877-898$.

7. Золотарев B. М. Об одной вероятностной задаче. - Теория вероятн. и ее примен., 1961, т. VI, в. 2, с. 219-222.

8. Richter W.-D. Gaußsche Wahrscheinlichkeiten großer Abweichungen im Banachraum $l_{p} .-$ Wissensch. Z. Techn. Univ. Dresden, 1985, v. 34, № 4, p. 60.

9. Dobric V., Marcus $M$. B., Weber M. The distribution of large values of the supremum of Gaussian processes. - Astérisque, 1988, v. 157-158, p. 95-127.

10. Fatalov V. R., Richter $W .-D$. Gaussian probabilities of large deviation for fixed or increasing dimension. - J. Contemporary Math. Analysis (Armenian Acad. Sci.), 1992 , v. 27, № 1, p. 1-16.

11. Fatalov $V . R$. Exact asymptotics of large deviations for Gaussian measures on Hilbert space. - J. Contemporary Math. Analysis (Armenian Acad. Sci.), 1992, v. 27, № 5, p. 36-50.

12. Fatalov $V . R$. Asymptotics of large deviation probabilities for Gaussian fields, I, II. J. Contemporary Math. Analysis (Armenian Acad. Sci.), 1992, v. 27, № 6, p. 48-70.

13. Го X.-С. Гауссовские меры в банаховых пространствах. М.: Мир, 1979, 176 с.

14. Chevet S. Gaussian measures and large deviations. - Lect. Notes Math., 1983, v. 990. p. 30-46.

15. Ellis R. S., Rosen J. S. Asymptotic analysis of Gaussian integrals, I: Isolated minimum points. - Trans. Amer. Math. Soc., 1982, v. 273, № 2, p. 447-481.

16. Вентиель А. Д. Предельные теоремы о больших уклонениях для марковских случайных процессов. М.: Наука, 1986, 176 с.

17. Цирельсоя Б. С. Плотность распределения махсимума гауссовского процесса. Теория вероятн. и ее примен., 1975, т. XX, в. 4, с. 865-873.

18. Rajput $B$. $S$. Gaussian measures on $L_{p}$ spaces, $1 \leqslant p<\infty$. - J. Multivariate Anal., 1972 , v. 2, № 4, p. 382-403.

19. Пруднихов А. П., Брычков Ю. А., Маричев О. И. Интегралы и ряды. Элементарные функции. М.: Наука, 1981, 800 c.

20. Абрамович М., Стиган И. (ред.) Справочник по специальным функциям. М.: Наука, 1979, 832 c. 
21. Бейтмея K., Эрдейи A. Высшие трансцендентные функции, т. 1: Гипергеометрическая функция, функции Лежандра. М.: Наука, 1965, 296 с.

22. Биллинесли П. Сходимость вероятностных мер. М.: Наука, 1977, 352 с.

23. Норвайиа $P$. Центральная предельная теорема для эмпирических и квантильных процессов в пространствах $L_{p}, 1 \leqslant p<\infty$. - Теория вероятн. и ее примен., 1992, т. 37 , в. 2 , с. $329-341$.

24. Лифиии $M . A$. Гауссовские большие уклонения гладкой полунормы. - Записки научн. семинаров ПОМИ, 1992, т. 194, с. 106-113.

25. Linde $W$. Gaussian measures of large balls in $l^{p}$. - Ann. Probab., 1991, v. 19, № 3, p. $1264-1279$.

26. Linde W. Gaussian measures of large balls in $\mathbf{R}^{n}$. - In: Stable Processes and Related Topics. Basel-Boston: Birkhaüser, 1991, p. 1-25.

27. Richter W.-D. Multidimensional domains of large deviations. - In: Limit Theorems in Probability and Statistics (Colloquia Mathematica Societas János Bolyai, v. 57). Amsterdam, 1990, p. 443-458.

Поступила в редакцию

25.VI.1993

Исправленный вариант

20.VII.1995

(c) $1996 \mathrm{r}$.

ЦИГРОШВИЛИ 3. П.*

\title{
О МОДУЛЯХ НЕПРЕРЫВНОСТИ ОБНОВЛЯЮЩИХ РАЗВЕРТОК ЭМПИРИЧЕСКИХ ПОЛЕЙ
}

\begin{abstract}
В статье исследуется асимптотическое поведение модуля непрерывности обновляющи разверток эмпирических полей, которые, в отличие от эмпирических полей, имеют некоррелированные прирацения. Доказывается, что модули непрерывности обновляющих разверток асимптотически ведут себя так же, как модули непрерывности эмпирических полей.
\end{abstract}

Ключевые слова и Фразы: эмпирические поля, обновляюшие развертки, модуль непрерывности.

1. Введение. Как известно из общей теории сходимости случайных процессов в разных функциональных пространствах (например, $C[0,1]^{d}, D[0,1]^{d}, d \geqslant 1$ ), сходимость последовательности случайных процессов $\xi_{n}$ к случайному прощессу $\xi$ доказывается в два этапа: а) сходимость конечномерных распределений процесса $\xi_{n}$; б) относительная компактность последовательности распределений $\xi_{n}$ (см. [1]).

Тогда как часть а) является, как правило, следствием центральной предельной теоремы, доказывать часть б) гораздо сложнее. Здесь надо показать, что для произвольных $\varepsilon>0$ и $\eta>0$ существует такое $a>0$, что для достаточно больших $n$ вытолняется неравенство $\mathbf{P}\left\{\omega_{n}^{\xi}(a) \geqslant \varepsilon\right\} \leqslant \eta$, где $\omega_{n}^{\xi}(a)=\sup _{|t-8| \leqslant a}\left|\xi_{n}(t)-\xi_{n}(s)\right|$ есть модуль непрерывности процесса $\xi_{n}$. Исследованием модуля непрерывности разных эмпирических процессов и полей занимались многие авторы (см., например, [2], [5]-[7]).

*Математический институт им. А. Размадзе АН Грузии, ул. Алексидзе, 1, 380093 Тбилиси, Грузия. 